\title{
Thermal conductivity measurements and self-organized criticality very close to the superfluid transition in ${ }^{4} \mathrm{He}$
}

\author{
W. A. Moeur, P. K. Day, F.-C. Liu, and R. V. Duncan \\ University of New Mexico, New Mexico, USA \\ E-mail: duncan@coffee.phys.unm.edu
}

We would like tp present the results of thermal conductivity measurements in ${ }^{4} \mathrm{He}$ very close to the superfluid transition in a range of heat flux $5 \mathrm{nW} / \mathrm{cm}^{2}<Q<100 \mathrm{nW} / \mathrm{cm}^{2}$. These measurements were performed in an experimental cell which employs a unique side-wall probe geometry. These side-wall probes allow us to circumvent the boundary effects usually associated with measurements made with end cap thermometer probes. The side-wall probes also facilitated a single probe measurement technique that allowed measurements of thermal conductivity to within several nanoKelvin of the superfluid transition temperature. In this region of temperatures, the thermal conductivity is predicted to display a dependence on the heat flux applied.

In addition to the thermal conductivity measurements, we report on an experimental observation of self-organized criticality in ${ }^{4} \mathrm{He}$ very close to its superfluid transition. A constant temperature gradient, independent of the heat flux $Q$ through the sample, is created along a vertical column of ${ }^{4} \mathrm{He}$ by applying heat to the top of the column. This constant temperature gradient equals the gravity-induced gradient in the superfluid transition temperature, indicating that the thermal conductivity of the sample has self-organized. The closeness to criticality in this state is the same throughout most of the sample, and it depends only on $Q$. These measurements have been made in a range of $Q$ from 0.04 to $6.5 \mathrm{~mW} / \mathrm{cm}^{2}$ in the absence of convection.

This work was accomplished with support from the NASA Microgravity Science and Applications Division as part of the DYNAMX flight project. This project intends to measure the thermal conductivity of ${ }^{4} \mathrm{He}$ close to the superfluid transition in a microgravity environment aboard a NASA space shuttle. 